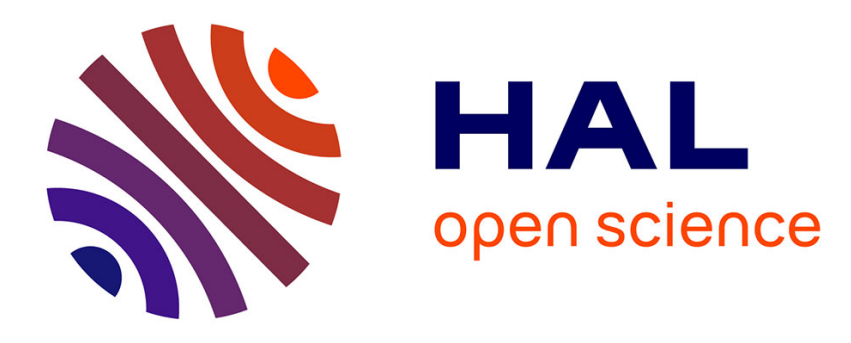

\title{
Numerical study of nonlinear elastic behaviour of structurally inhomogeneous media
}

\author{
A. Bogdanov
}

\section{To cite this version:}

A. Bogdanov. Numerical study of nonlinear elastic behaviour of structurally inhomogeneous media. Journal de Physique IV Proceedings, 1994, 04 (C5), pp.C5-697-C5-700. 10.1051/jp4:19945149 . jpa00252827

\section{HAL Id: jpa-00252827 https://hal.science/jpa-00252827}

Submitted on 1 Jan 1994

HAL is a multi-disciplinary open access archive for the deposit and dissemination of scientific research documents, whether they are published or not. The documents may come from teaching and research institutions in France or abroad, or from public or private research centers.
L'archive ouverte pluridisciplinaire HAL, est destinée au dépôt et à la diffusion de documents scientifiques de niveau recherche, publiés ou non, émanant des établissements d'enseignement et de recherche français ou étrangers, des laboratoires publics ou privés. 


\title{
Numerical study of nonlinear elastic behaviour of structurally inhomogeneous media
}

\section{A.N. BOGDANOV}

N.N. Andreev Acoustic Institute, Russian Academy of Sciences, Shvernik st. 4, 117036 Moscow, Russia

\begin{abstract}
Nonlinear elastic properties of structurally inhomogeneous media are investigated in numerical experiment. For these media to be simulated, the lattices of masses (sites), connected by nonlinear springs, are used. For the cases of 2D triangular and 3D fcc lattices, the dependence of linear and nonlinear elastic modules and nonlinearity parameters of second and third orders on the number of defects (absent sites) is obtained. Scaling behaviour of nonlinear elastic modules is studied in the vicinity of percolation threshold ( $i . e$. near the point, where all elastic modules vanish).
\end{abstract}

\section{INTRODUCTION}

At present, special attention is assigned to investigations of the media with structural inner inhomogeneities [1-3] (granular, porous, cracked and others), because they can be characterized by great nonlinearity parameter (several orders higher than that of a homogeneous material). In present paper, the results of numerical study of nonlinear elasicic properties of structurally inhomogeneous materials are reported and discussed, with the model of the media is taken in realistic form, when certain part of structural elements (grains) is removed at random. This model is treated as an elastic percolation system and, in practice, corresponds to the unconsolidated inhomogeneous rocks and to the forming of branched net of cracks in the initially uniform sample.

\section{METHOD AND PROCEDURE}

The basic model of structurally inhomogeneous medium is represented by the lattice, which is consisted of masses (sites) 
connected with springs. The calculations have been carried out for the $2 D$ triangular lattice and fcc lattice in 3D case. The potential energy of interaction between the elements (energy of spring deformation) was chosen in the form:

$U=\frac{\alpha}{2}(\mathrm{~d} r)^{2}+\frac{\beta}{n+1}(\mathrm{~d} r)^{\mathrm{n}+1}$

where $\alpha, \beta=$ const, $d r$ was deformation of the spring and $n$ was the nonlinearity parameter. The expression (1) corresponds to the nonlinear force of interaction between the sites $F=-\partial U / \partial(\mathrm{d} r)$ and can be used as the simple model of contact interaction between the grains in unconsolidated rocks [4]. The parameters of the problem $\alpha$, $\beta$ were chosen, so that physical nonlinearity (nonlinearity of Hook's law) was considerably larger than geometrical nonlinearity of the medium.

The simulation of structurally inhomogeneous material was done with the help of well-known Monte-Carlo method [5], in which certain part of sites $q=1-p$ was removed from the lattice at random ( $p$ was the probability of a site presence). In given computer experiment the triangular lattice of $30 \times 30=900$ sites was modeled in $2 \mathrm{D}$ case and the fcc lattice of 1099 sites - in 3D case. When the obtained depleted lattice had been deformed in a specific way (uniform compression, linear compression or pure shear), the usual relaxation method [5] was applied in order to lead the system to new equilibrium position. For the nonlinear elastic modules to be defined the expansion of the stress tensor component $\sigma_{i j}$ by the degrees of strain tensor $u_{i j}$ was used.

The deformations of compression and shear were investigated separately, which gave us the opportunity to find the dependence of the elastic modules on $p$. In the present work only quadratic $(n=2)$ and cubical $(n=3)$ nonlinearities were considered (and, respectively, the modules $k_{1}, \quad k_{2}, k_{3}$ (uniform compression), $c_{1}, c_{2}, c_{3}$ (linear compression) and $\mu_{1}, \mu_{2}, \mu_{3}$ (pure shear), with $\mu_{2}$ being null for both cases, because of the symmetry of the lattices).

\section{RESULTS.}

The examples of calculated dependence of the elastic modules on $p$ are shown in the fig. 1 (uniform compression $-k_{1}, k_{2}, k_{3}$ ) and $f i g .2$ (shear $-\mu_{1}, \mu_{3}$ ) for fcc net. The vertical axis measures the magnitudes of the elastic modules $K_{1}, K_{2}, K_{3}$ and $\mu_{1}, \mu_{3}$, related to the respective values for the medium without defects $p=1 \%$ $\overline{K_{1}}(p)=K_{1}(p) / K_{1}(1)$ and so on. The value of percolation concentration 

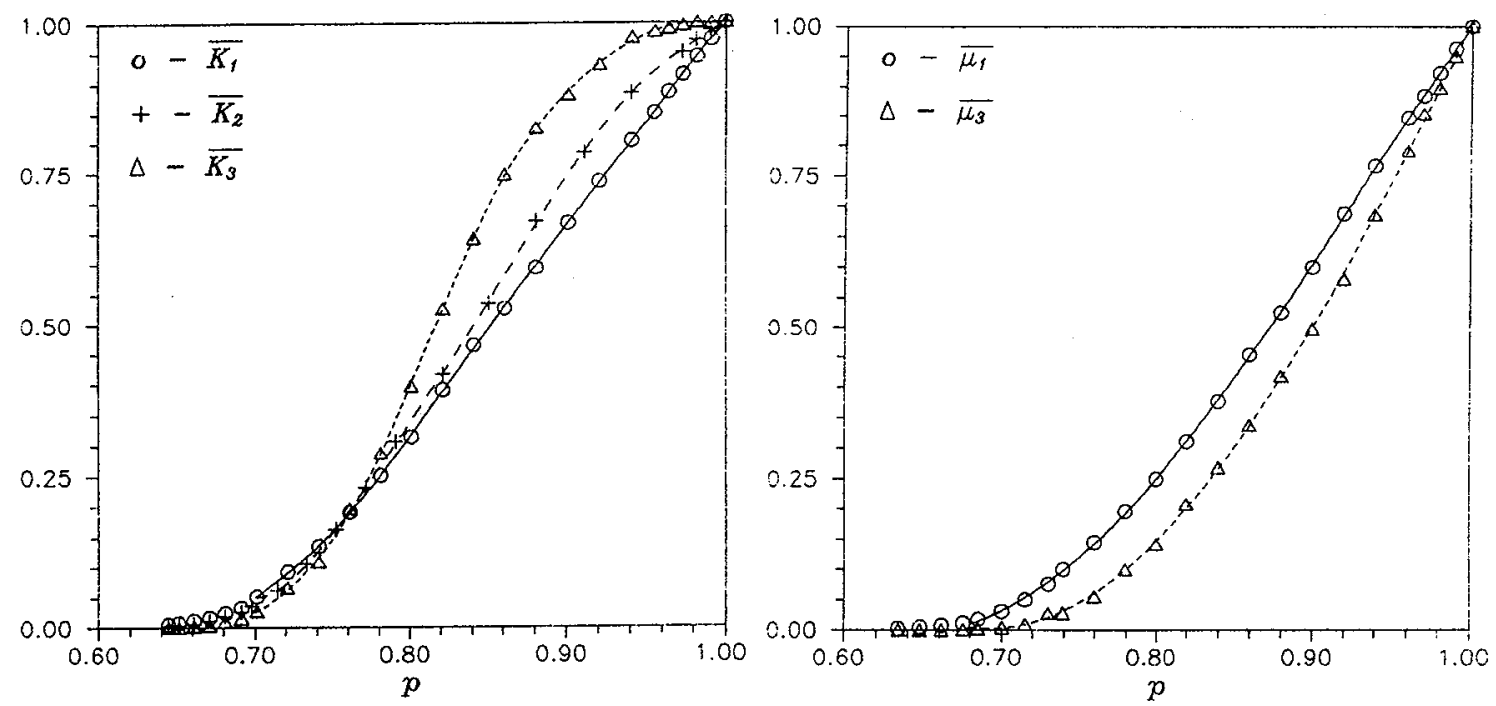

Fig. 1. Dependence of elastic modules of uniform

Fig.2. Dependence of elastic compression on $p$.

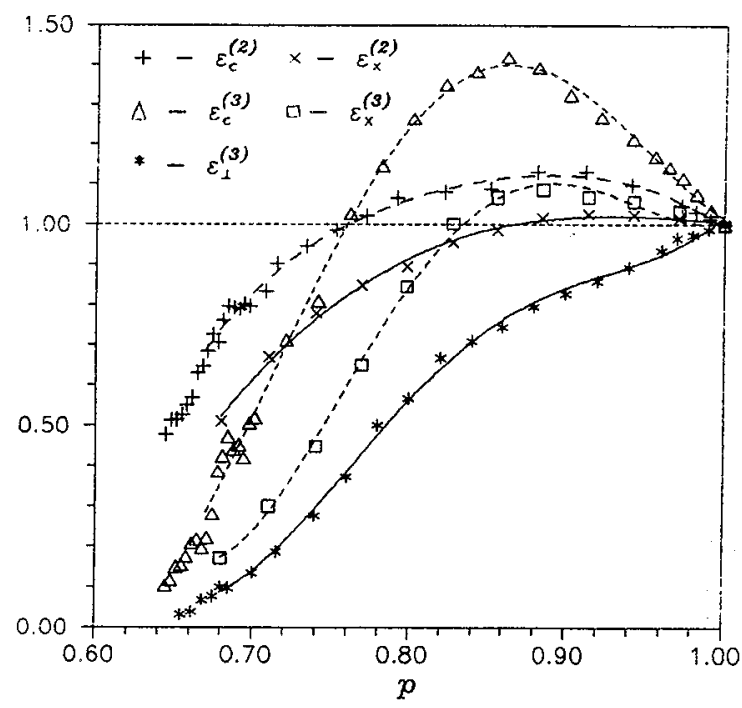

Fig.3. Nonlinearity parameters of 2-nd and 3-rd orders for uniform compression, innear compression and shear. 
$p=p^{*}$, where all the modules vanish, is referred to as percolation threshold [5]. In our calculation, the appropriate thresholds $p^{*}=0.59 \pm 0.01$ and $p^{*}=0.42 \pm 0.01$ have been obtained. In the vicinity of percolation threshold the dependence of all elastic modules on $p$ has scaling character, i.e. their magnitude is proportional to $\left(p-p^{*}\right)^{v_{n}}$, where $v_{n}$ is the percolation exponent, determined by the dimension of the system $d$ and by the order nonlinearity $n$ (see (1)). Analysis of our results demonstrates the presence of common relation $v_{1}<v_{2}<v_{3}$, where $v_{1}$ is the exponent, corresponding to the modules $K_{1}, c_{1}, \mu_{1} ; v_{2}$ to $K_{2}, C_{2} ; \quad v_{3}$ to $K_{3}, C_{3}, \mu_{3}$. For instance, $v_{1}=2.6 \pm 0.5, v_{2}=3.3 \pm 0.7$, $v_{3}=4.3 \pm 1.0$ for triangular lattice and $v_{1}=3.4 \pm 0.6, \quad v_{2}=4.0 \pm 1.0$, $5.5<v_{3}<8.5$ for fcc lattice.

The figure 3 demonstrates the dependence of $\varepsilon_{c}^{(2)}=\overline{K_{2}}(p) / \overline{K_{1}}(p)$, $\varepsilon_{c}^{(3)}=\overline{K_{3}}(p) / \overline{K_{1}}(p), \quad \varepsilon_{\mathrm{x}}^{(2)}=\overline{C_{2}}(p) / \overline{C_{1}}(p), \quad \varepsilon_{\mathrm{x}}^{(3)}=\overline{C_{3}}(p) / \overline{C_{1}}(p), \quad \varepsilon_{\perp}^{(3)}=\overline{\mu_{3}}(p) / \overline{\mu_{1}}(p)$ on percolation concentration $p$. These relations have the sense of nonlinearity parameters, which are widely used in acoustics [1]. It is important to notice, that the dependence of nonlinearity parameters $\varepsilon_{c}^{(2)}, \varepsilon_{c}^{(3)}$ on $p$ has not monotonous character in the wide range of concentration $(0.75 \leq p<1$ in $2 \mathrm{D}$ case and $0.6 \leq p<1$ in $3 \mathrm{D}$ case), in which the parameters exceed unity. The parameter $\varepsilon_{\perp}^{(3)}$ always decreases and comes to zero at $p^{*}$.

It can be proposed, that the features of nonlinear behaviour of structurally inhomogeneous material, mentioned above, have sufficiently common character and retain (although qualitatively) in the transition to more complex models of the media (anisotropic, media with noncentral forces etc. $[4,6,7]$ ).

\section{REFERENCES}

[1] Rudenko 0.V. and Soluyan S.I., Theoretical foundations of nonlinear acoustics (Consultants Bureau, New York, 1977).

[2] Erofeev V.I. and Potapov A.I., in: Nonlinear World 1989 V.2 (IV Int. Workshop on Nonlinear and Turbulent Processes in Physics, 1989) pp.1197-1215.

[3] Berryman J.G., J. Acoust. Soc. Am. 68 (1980) 1809-1831.

4] Schwartz L.M., Feng S., Thorpe M.F., Sen P.N., Phys. Rev. B 32 (1985) $4607-4617$.

5] Feng S. and Sen P.N., Phys. Rev. Lett. 52 (1984) 216-219.

6. Feng S., Phys. Rev. B 32 (1985) 510-513.

7) Tang W. and Thorpe M.F., Phys. Rev. B 37 (1988) 5539-5551. 